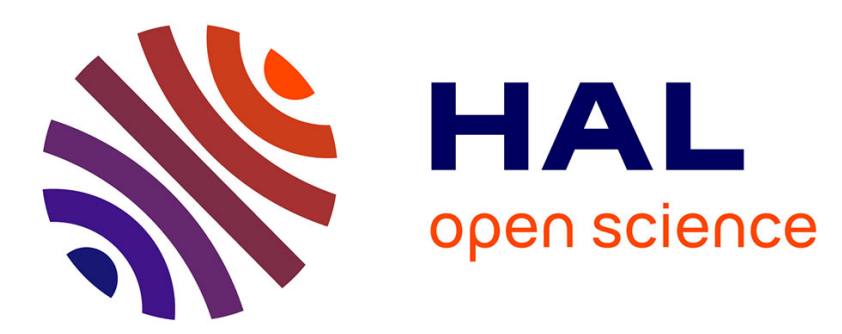

\title{
La coupellation des alliages cuivre-argent : approche expérimentale de l'essai d'argent par voie sèche.
}

Florian Téreygeol, Nicolas Thomas

\section{To cite this version:}

Florian Téreygeol, Nicolas Thomas. La coupellation des alliages cuivre-argent: approche expérimentale de l'essai d'argent par voie sèche.. Archeosciences, revue d'Archéométrie, 2003, 27, pp.171 - 181. 10.3406/arsci.2003.1053 . hal-01766865

\section{HAL Id: hal-01766865 https://hal-inrap.archives-ouvertes.fr/hal-01766865}

Submitted on 14 Apr 2018

HAL is a multi-disciplinary open access archive for the deposit and dissemination of scientific research documents, whether they are published or not. The documents may come from teaching and research institutions in France or abroad, or from public or private research centers.
L'archive ouverte pluridisciplinaire HAL, est destinée au dépôt et à la diffusion de documents scientifiques de niveau recherche, publiés ou non, émanant des établissements d'enseignement et de recherche français ou étrangers, des laboratoires publics ou privés. 


\title{
LA COUPELLATION DES ALLIAGES CUIVRE - ARGENT : APPROCHE EXPÉRIMENTALE DE L'ESSAI D'ARGENT PAR VOIE SÈCHE
}

\author{
Florian TÉREYGEOL* et Nicolas THOMAS**
}

\begin{abstract}
Résumé : La définition du titre d'un alliage dans le cadre d'une production monétaire nécessite une grande rigueur car c'est sur cette opération que repose l'acceptation ou non d'un lot de monnaies. Une des méthodes employées pour y parvenir est l'essai d'argent par voie sèche. Il s'agit de séparer le métal blanc du plomb et du cuivre par oxydation de ces derniers. S'il est relativement aisé de séparer le plomb de l'argent, la coupellation du cuivre nécessite une plus grande maîtrise technique. Les données d'archive fournissent la marche à suivre dans un cadre expérimental. La difficulté réside dans l'ajout nécessaire de plomb pour permettre la séparation du cuivre. La quantité à ajouter varie de 3 à 17 fois la masse de cuivre estimée. Un fourneau d'essayeur a été bâti à Melle, et une série d'alliages Cu-Ag réalisée in situ a pu être essayée. Le four est appareillé afin de contrôler la température. Pour estimer la qualité de l'opération exécutée, les boutons de retour sont tout d'abord pesés puis ils font l'objet d'une analyse quantitative. Les coupelles ayant servi à ces opérations sont aussi observées car elles permettent de recueillir des informations facilitant l'analyse et l'interprétation des artefacts archéologiques.
\end{abstract}

\begin{abstract}
The quality of the alloy used was an essential factor in the acceptance or rejection of coins. One test method used was fire assaying. This consists of separating the silver from both the lead and the copper which are oxidised during the process. If it is fairly easy to separate lead from silver, copper cupellation requires considerable skill. Documentary sources provide valuable guidance on how to carry out an experiment, the principal difficulty being the amount of lead required for successful copper separation. The quantity proposed varies between three and seventeen times the estimated amount of copper used. A trial furnace, equipped with temperature control, was set up in Melle and a set of $\mathrm{Cu}-\mathrm{Ag}$ alloys was tested. In order to judge the quality of the experiment, the silver droplets were weighed and then analysed. The cupels were also examined so as to maximise the information available for the future analysis and interpretation of archaeological artefacts.
\end{abstract}

Mots-clés : Coupellation, argent, cuivre, expérimentation.

Key-words : Cupellation, silver, copper, archaeological experiment.

\section{INTRODUCTION}

Dans l'histoire monétaire médiévale et moderne, l'or et l'argent occupent une place prépondérante, la valeur d'une monnaie étant garantie par la quantité de métal précieux qu'elle contient. Cette position privilégiée tendrait à faire oublier la présence d'un troisième métal : le cuivre. Ce dernier est tout aussi important. Il est allié avec les deux premiers afin de préparer la matière première de la production monétaire. Le cuivre est également lié à l'argent dans plusieurs minerais complexes et riches en métal blanc. Les métallurgistes, les alchimistes et les essayeurs ont dû adapter une méthode permettant le dosage de ces deux métaux lorsqu'ils se trouvent associés : la coupellation. Cette technique repose sur la plus grande facilité à oxyder en phase liquide le plomb, mais aussi le cuivre, alors que le métal précieux reste à l'état métallique. Ainsi les essais d'alliages cuivre-argent sont fondés sur la purification de l'argent par coupellation avec ajout de plomb et sur un dosage du métal précieux par différence. Il faut peser la prise au départ, puis peser le métal précieux restant au terme de l'opération. Par une simple règle de trois, on obtient le titre en métal blanc alors que la quantité de cuivre est connue par soustraction. L'oxyde de cuivre seul est trop difficilement fusible dans les types de fours utilisés. Il faut pratiquer lors de la coupellation un emplombage permettant d'abaisser le point de fusion du mélange. L'essai sur un alliage binaire argent/cuivre implique en définitive trois métaux : l'argent, le cuivre et le plomb. Le procédé de coupellation en lui-même ne varie pas que l'on travaille ou non du cuivre avec le plomb et l'argent. Il consiste toujours, en phase liquide, 
à oxyder le bain à la surface duquel se forme une couche d'oxydes provoquant un enrichissement du bain métallique en argent. Toute la difficulté réside dans l'évacuation progressive de ces oxydes sans quoi l'opération ne peut aboutir. C'est pourquoi, dans le cadre d'un essai, la coupellation est pratiquée dans un réfractaire poreux fabriqué essentiellement avec de la cendre d'os. D'autres matériaux poreux sont connus comme la cendre de bois mais la cendre d'os semble avoir la préférence des essayeurs. La coupelle va peu à peu s'imbiber d'oxydes laissant à terme un bouton d'argent. La coupelle de cendre absorbe les oxydes en phase liquide mais pas les métaux. Cette propriété est la conséquence de tensions superficielles différentes des deux liquides : un oxyde en phase liquide « mouille » plus qu'un métal dans le même état.

Ce mode de purification à hautes températures est très ancien. Il est décrit dès l'Antiquité dans les textes grecs, puis dans des textes arabes et ensuite dans les plus anciens traités techniques et alchimiques de 1'Occident médiéval (Toll, 1968 ; Caunedo \& Cordoba, 2000 ; Clairand et Kind, 2000).

Les plus anciennes recettes de coupellation demeurent très laconiques comme dans le Papyrus de Leyde (III-IVe siècles ap. J.-C.) : «Rendre tout argent pur et brillant. Prenez une partie d'argent et la même quantité de plomb, laissez fondre au four jusqu'à ce que le plomb soit consumé. Faites cela souvent jusqu'à ce qu'il devienne brillant » (Halleux, 1981).

L'utilisation de ce procédé comme méthode d'essai et d'analyse devient manifeste en 1343 avec l'ordonnance de Philippe de Valois adressée aux essayeurs décrivant avec précision l'opération (Hoefer, 1866-1869). Si la monnaie est un fait du prince destiné à asseoir son pouvoir tant économique que politique, il ne serait rien sans une méthode d'analyse fiable et universellement diffusée.

Les données écrites sur l'utilisation de ce mode opératoire pour un alliage contenant de l'argent et du cuivre sont plus précises dans les traités métallurgiques du XVI siècle :

«Les alliages de métaux que l'on appelle monétaires, sont essayés de la manière suivante $[. .$.$] on prend huit$ onces de granulés, ou un poids égal de copeaux et encore le même poids. [...] on met deux petits morceaux de plomb dans les coupelles qui ont d'abord été chauffées. Plus la monnaie est précieuse, moins il faut de plomb pour 1'essai ; plus elle est vile, plus il en faut. Pour huit onces d'argent, que l'on sait contenir seulement une demi-once ou une once de cuivre, nous ajoutons aux huit onces de granulés une demi-once de plomb. Si elle est composée de parties égales d'argent ou de cuivre, nous ajoutons une demi-once de plomb. Mais dans huit onces de cuivre, il y a seulement une once ou une demi-once d'argent, il faut une once et demi de plomb » (Agricola, 1987).

Les textes précédant le Diversarum artium schedula, ou Essai sur divers arts du moine Théophile (XII ${ }^{\mathrm{e}}$ siècle), sont totalement muets sur la nature du contenant employé (Théophile, 1977). Dès le XIII ${ }^{\mathrm{e}}$ siècle, la composition de la coupelle donne le nom à la technique d'essai, celle-ci étant appelée cineritium ou « cendrée » par l'alchimiste
pseudo-Geber (Newman, 1991). Le terme cupella pour désigner le contenant et le nom de l'opération n'est probablement pas antérieur au XVe siècle (Corbett, 1939).

Les découvertes archéologiques de coupelles de cendre sont encore peu nombreuses. Une belle collection provenant de l'atelier monétaire de Pymont (Jura) et datant du XIV` siècle est connue (Jeanjacquot et Goy, 1993). Quatre autres exemplaires ont été retrouvés lors des fouilles de l'atelier monétaire de La Rochelle (Charente-Maritime). En Allemagne, il existe plusieurs coupelles conservées au musée de Bochum mais dont l'origine reste inconnue (Slotta et al., 1999). Au total, sur l'ensemble de l'Europe, une trentaine d'exemplaires a pu être identifiée. Leurs datations s'échelonnent du XIV siècle au XVIII ${ }^{e}$ siècle (Rehren, 1996, 1997 et 1998 ; Goldenberg et Wagner, 1994 ; Goy et Thomas, 2003).

\section{OBJECTIFS}

L'approche expérimentale de l'essai des alliages cuivre-argent par la voie sèche poursuit trois objectifs distincts. Une première série d'essais avait été conduite avec succès sur des alliages $\mathrm{Pb}-\mathrm{Ag}$ (Téreygeol et Thomas, à paraître). L'essai sur des alliages difficiles à fondre mais utilisés communément pour la frappe monétaire est donc une suite logique du travail sur l'essai d'argent par voie sèche. En effet, durant l'opération, il faut atteindre le point de fusion de l'alliage (de $775^{\circ} \mathrm{C}$ à $1000^{\circ} \mathrm{C}$ ). Il est surtout important de parvenir à la température de fusion des oxydes. Pour l'oxyde de plomb, elle est de $860^{\circ} \mathrm{C}$ alors que pour l'oxyde de cuivre, elle se trouve à plus de $1200^{\circ} \mathrm{C}$. En suivant le diagramme d'équilibre ( $\mathrm{PbO}-$ $\mathrm{CuO}$ ), on comprend alors clairement l'importance de

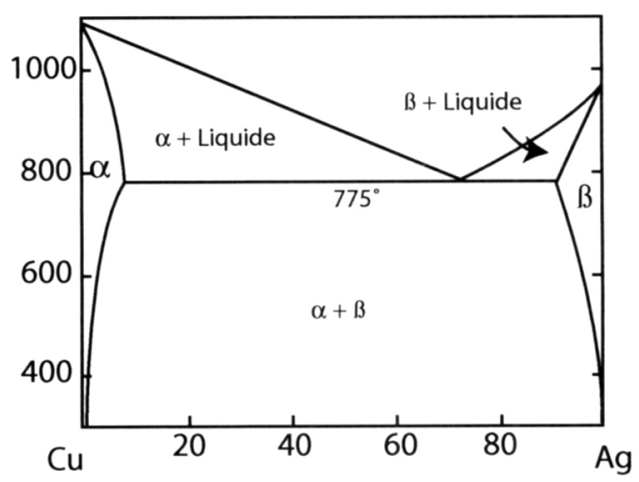

Système Cu-Ag

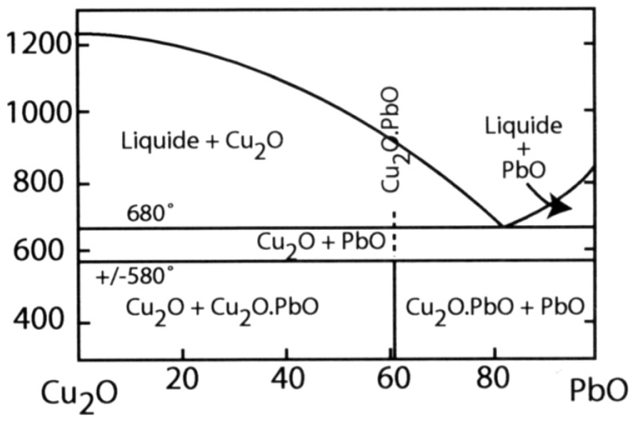

Système $\mathrm{Cu}_{2} \mathrm{O}-\mathrm{PbO}$

Fig. 1 : Diagrammes de phase $\mathrm{Cu}-\mathrm{Ag}$ et $\mathrm{Cu}_{2} \mathrm{O}-\mathrm{PbO}$, d'après Gebhardt E. et Obrowski, W. Z. Metall., 45, (1954). 
l'ajout de plomb en quantité. En particulier, le diagramme de phase indique une température de fusion du mélange comprise entre 800 et $860^{\circ} \mathrm{C}$ dès lors que le mélange contient plus de $95 \%$ de plomb (fig. 1). Quelque soit le rapport des oxydes, si la température vient à chuter en deçà de $680^{\circ} \mathrm{C}$, ils passent en phase solide entraînant l'ennoyage de l'essai et son échec. Entre ce point et la température de fusion, pour une composition supérieur à $95 \%$ de $\mathrm{PbO}$, deux phases co-existent : le bain métallique $\mathrm{Pb}-\mathrm{Ag}-\mathrm{Cu}$ et le mélange liquide $\mathrm{Cu} 2 \mathrm{O}-\mathrm{PbO}$. Les essayeurs se sont appliqués à toujours maintenir un rapport $\mathrm{Cu} 2 \mathrm{O}-\mathrm{PbO}$ supérieur à $95 \%$ de $\mathrm{PbO}$ afin de préserver une marge de sécurité entre la phase liquide et le passage à l'état solide. Ils n'ont pas cherché à travailler au voisinage de l'eutectique $\mathrm{Cu} 2 \mathrm{O}-\mathrm{PbO}$ pour deux raisons. La phase liquide est par définition trop proche de la phase solide. D'autre part, si pour une raison de mauvais dosage le taux de $\mathrm{PbO}$ venait à être inférieur à $80 \%$, une baisse de température en dessous du liquidus donnerait un liquide contenant comme phase solide non plus $\mathrm{PbO}$ mais $\mathrm{Cu} 2 \mathrm{O}$. Une partie du cuivre n'est donc plus absorbée par la coupelle. Ce dosage précis du plomb tient aussi compte de la capacité d'absorption de la coupelle. Cette dernière est supposée pouvoir absorber sa masse en plomb (Riche et Gelis, 1888). Il est pourtant déconseillé d'ajouter inconsidérément du plomb car il en résulte un allongement de la durée de coupellation qui augmente la perte en argent (Berthier, 1834). Pendant les premiers essais, il n'avait pas été possible de mesurer les températures atteintes dans le four, les observations avaient été faites à partir des couleurs des parois. L'enregistrement de la température à l'intérieur du moufle durant l'opération est une aide précieuse pour maîtriser et comprendre le phénomène. Enfin, s'il existe une littérature relativement abondante sur les essais, les auteurs ne s'attardent jamais sur la coupelle après usage (Campredon, 1909). Or, il s'agit de l'artefact qui caractérise le mieux cette opération lorsqu'on le retrouve en fouille ${ }^{1}$. La création d'analogues expérimentaux est une alternative à l'indigence du mobilier découvert en fouille et au manque de description de cet objet après usage. Les coupelles expérimentales peuvent être étudiées sans contrainte de conservation pour ensuite être comparées aux artefacts archéologiques.

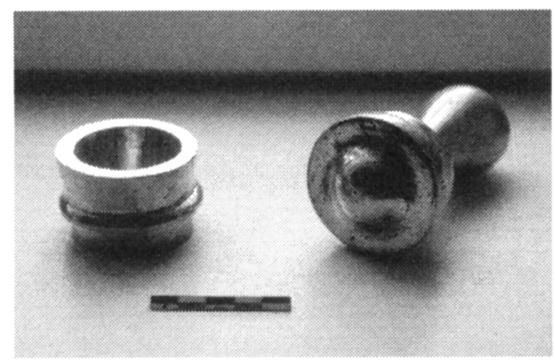

Fig. 2 : Le moule à coupelle.

\section{MONTAGE EXPÉRIMENTAL}

Cette expérimentation demande un matériel particulier qu'il a fallu recréer. Pour fabriquer les coupelles de cendre, il a fallu réaliser un moule en laiton ${ }^{2}$. Les dimensions et la forme des coupelles expérimentales correspondent aux exemples archéologiques du XIVe siècle. Le moule est composé de deux pièces : la " nonne " dans laquelle on place la cendre humidifiée, et le "moine ", sorte de pilon destiné à compresser la pâte et donner le volume intérieur de la coupelle (fig. 2). La plupart des recettes anciennes préconisent l'emploi de cendre d'os fortement calciné afin de ne conserver que les parties minérales de la matière. La préparation de cette cendre est longue et fastidieuse, c'est pourquoi certains textes mentionnent l'utilisation de cendre végétale plus aisée à produire. Dans le cadre de ces expérimentations, seule de la cendre d'os vendue dans le commerce a été utilisée. Cette cendre est mêlée à l'eau puis compactée dans le moule. La coupelle est ensuite mise à sécher lentement à l'ombre. Plus de 150 coupelles ont été ainsi moulées.

En ce qui concerne le four, on ne connaît pas les formes utilisées au Moyen Âge. Aucune découverte archéologique ne vient combler cette lacune. Les premiers textes décrivant ces fours précisément sont les traités de métallurgie du XVI ${ }^{\mathrm{e}}$ siècle comme les célèbres ouvrages de Biringuccio, De la pyrotechnia, et d'Agricola, De re metallica (fig. 3). Le four à essai possède une architecture particulière et fonctionne en tirage naturel, c'est-àdire qu'aucun soufflet ne vient alimenter le foyer en air. Il est composé de deux chambres imbriquées (fig. 4). La plus grande, ou chambre de combustion, est percée à sa base d'une multitude de trous autorisant à la fois la ventilation du foyer et l'évacuation des cendres. Au sommet, on trouve la cheminée d'évacuation des gaz de combustion. Le tirage se fait donc naturellement du bas vers le haut. La hauteur de la cheminée peut être réglable ce qui permet de contrôler le tirage et donc la température du four. Le chargement en charbon de bois se fait directement par la cheminée. À l'intérieur de cette chambre de combustion est placée une autre chambre appelée " moufle». Le moufle est une pièce d'argile réfractaire en forme de tunnel, ou demi-cylindre, qui est prise au milieu du foyer. Selon les modèles connus archéologiquement et en tenant compte des représentations iconographiques, ce moufle expérimental ne possède pas de sole. Il repose donc directement sur la sole de la chambre de chauffe qui, sur son emprise, n'est pas percée de trous de ventilation. Le moufle porte des petites ouvertures latérales ainsi qu'au fond du «tunnel ». Ces ouvertures favorisent la circulation d'air en permanence de l'extérieur du four vers l'intérieur du moufle puis vers la chambre de combustion. La coupelle et la prise d'essai sont introduites, à l'aide de pinces, dans le moufle où la température peut être facilement réglée en avançant plus ou moins le récipient dans le tunnel.

\footnotetext{
Des éléments de four et de moufle ont été retrouvés sur diverses fouilles mais ils ne peuvent être rattachés directement à l'essai pouvant servir à d'autres opérations métallurgiques comme la dessiccation et les actions de chauffage en général.

2 Ce travail a été confié à Jacques Happ.
} 
Les alliages ont également été réalisés sur le terrain en créant une gamme théorique partant d'un taux de $10 \%$ d'argent et croissant de $10 \%$ à chaque intervalle à l'exception du dernier alliage qui passe de $90 \%$ à $95 \%$ d'argent. Ces alliages ont été échantillonnés pour contrôler par analyse l'absence d'erreur lors de la pesée des composants. Il ne s'agissait pas de définir leur titre exact car ils ne sont pas homogènes ${ }^{3}$. Pour remédier à ce problème, Campredon préconise l'essai à la goutte, c'est-à-dire l'échantillonnage de l'alliage lorsque celui-ci est fondu. Les quantités produites expérimentalement n'ont pas permis de suivre ce conseil. Les prélèvements témoins ont eu lieu à froid. Les analyses des différents alliages montrent des taux souvent proches de ceux attendus. Les variations semblent être principalement le fait d'erreurs de pesée sur le terrain (fig. 5).

Au cours des expérimentations, des mesures de températures ont été faites grâce à des enregistreurs Gossen-Metrawatt équipés de thermocouples $\mathrm{K}$ permettant un contrôle continu des variations de température. Il faut

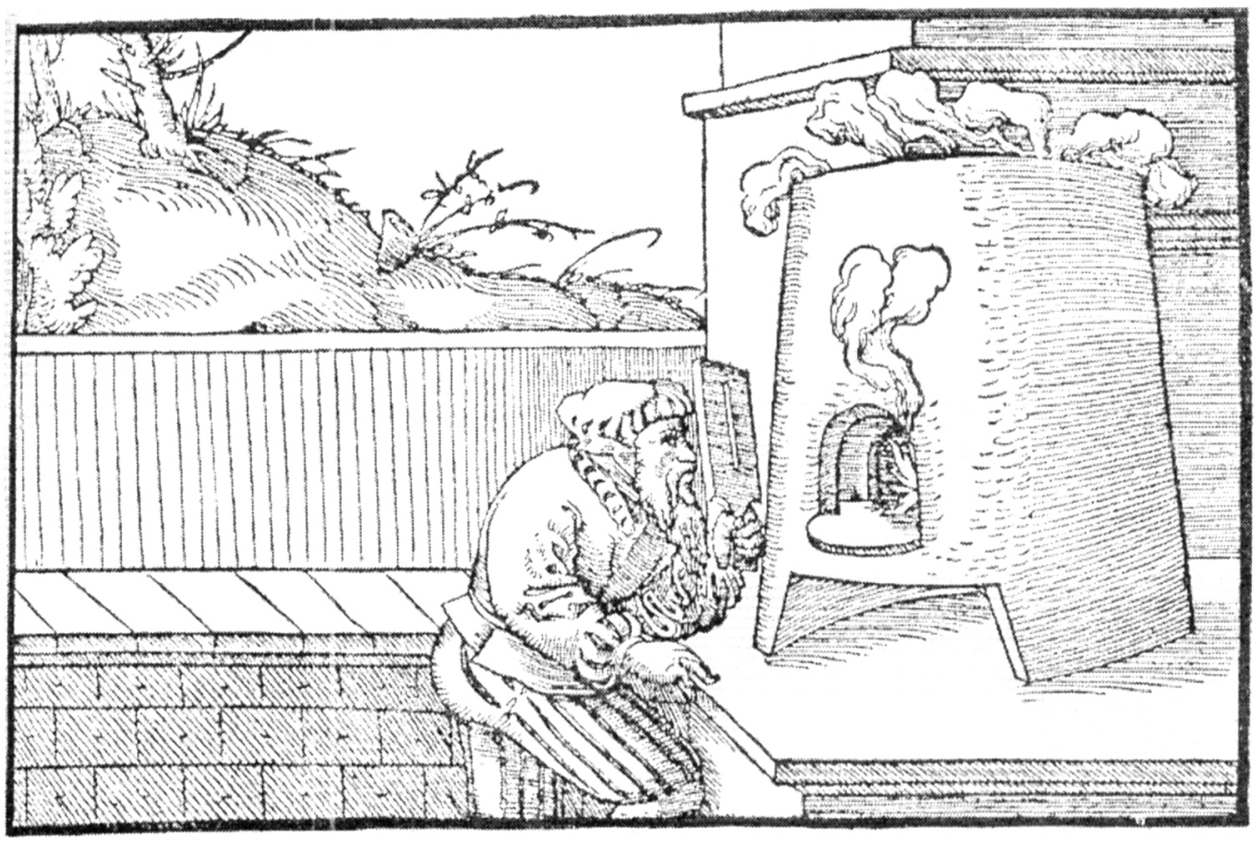

ROUND ASSAY FURNACE.

D'après Agricola, De Re metallica, 1556, ed. H.C.et L.H. Hoover 1912, ré-édition 1986, p. 223.

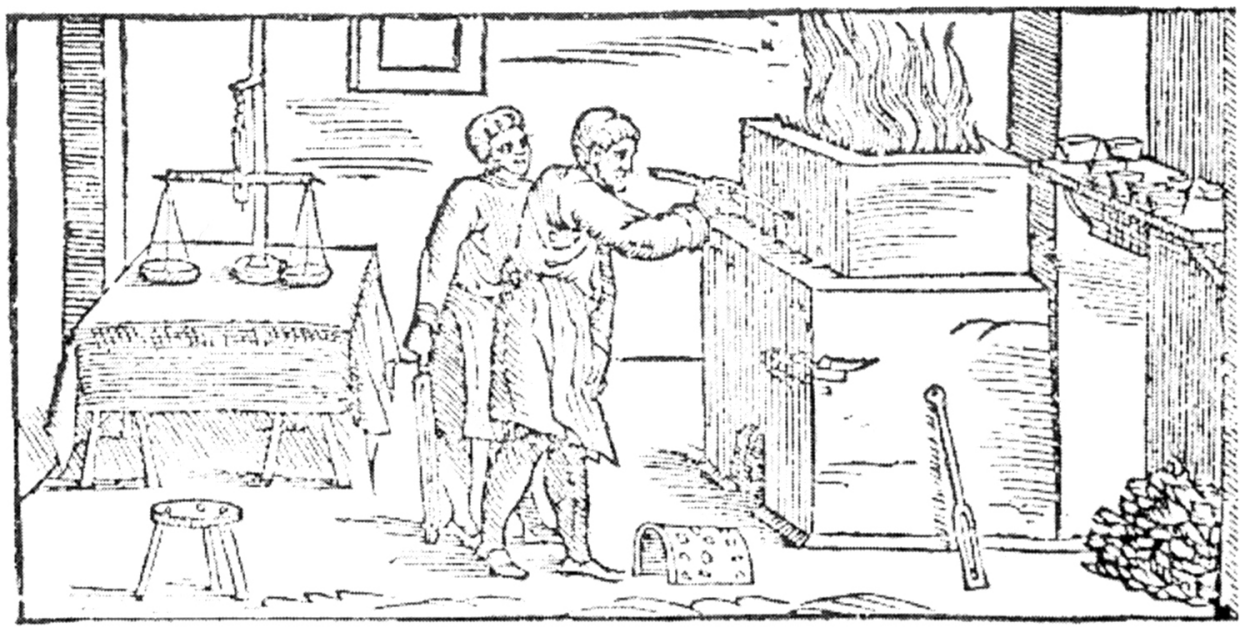

D'après Biringuccio, De la pirotechnia, 1540, ed.C.S. Stanley et M.T.Gnudi 1959, ré-édition 1990, p. 140.

Fig. 3 : Les fours à essai selon l'iconographie du XVI' siècle.

\footnotetext{
${ }^{3}$ Cette hétérogénéité avait déjà été remarquée, dès 1769 , par Jars qui observait que dans les alliages à bas titre le titre de la partie centrale est plus faible que celui de la périphérie. Bien plus tard, Campredon, dans son guide pratique du chimiste métallurgiste, art. 85, p. 34-35, fait les constatations suivantes :

« 1 Pour les alliages à 718.32 millièmes d'argent, correspondant à la formule $\mathrm{Ag} 3 \mathrm{Cu} 4$, il ne se produit pas de liquation [lors du refroidissement du lingot]; la teneur en argent est la même dans toutes les parties du lingot ;

2 Pour les alliages à teneur d'argent inférieur à 718.32, on trouve des teneurs d'argent plus faibles au centre qu'à la surface du lingot ;

3 Pour les alliages à teneur d'argent supérieure à 718.32, on trouve, au contraire, des teneurs d'argent plus fortes au centre qu'à la surface du lingot ».
} 
Coupe A

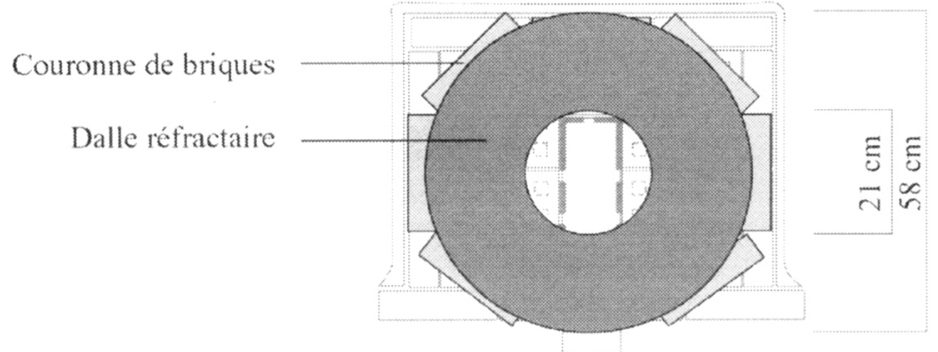

Coupe B

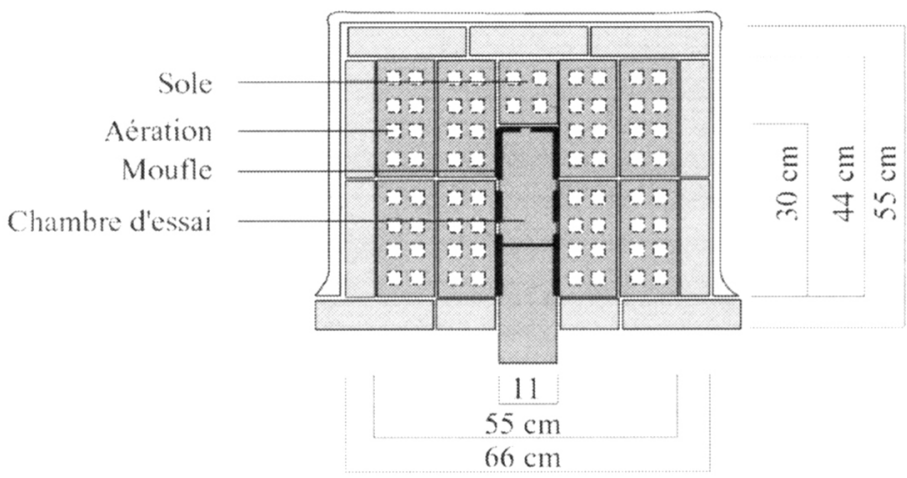

Vue de Face

Cheminée amovible

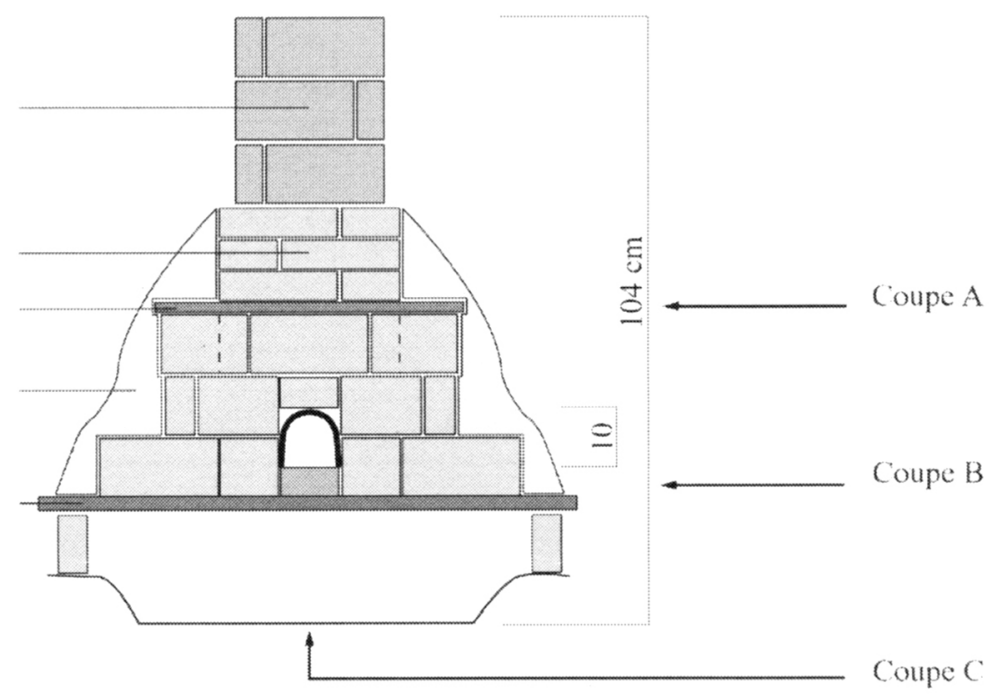

\section{Coupe C}

Chambre de chauffe

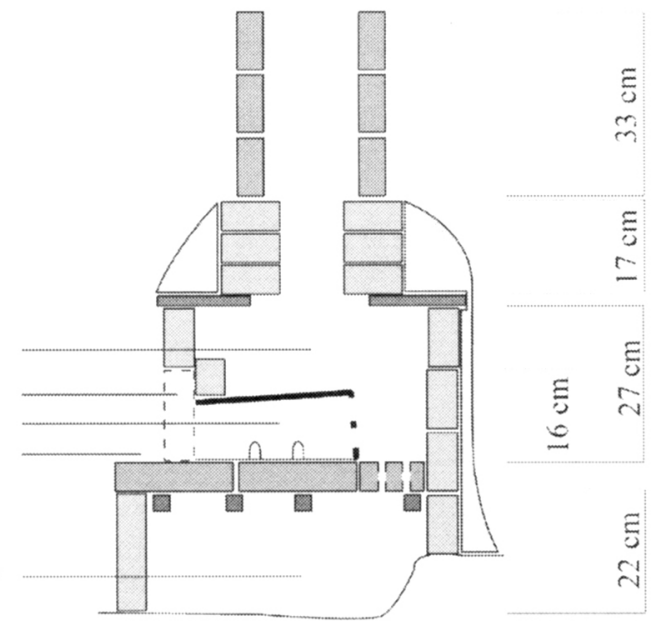

$30 \mathrm{~cm}$

$44 \mathrm{~cm}$
Porte amovible

Moufle et aérations

Pont de chargement

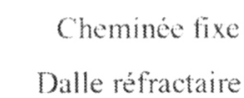

Revêtement d'argile

Barre de soutien

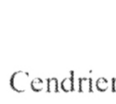

Four expérimental

d'essai de l'argent 


\begin{tabular}{|c|c|c|c|c|c|c|c|c|c|c|c|}
\hline $20 \mathrm{Ag}$ & & & & $30 \mathrm{Ag}$ & & & & $40 \mathrm{Ag}$ & & & \\
\hline Elt & \%mass & $\%$ mass & \%mass & Elt & \%mass & \%mass & \%mass & Elt & $\%$ mass & $\%$ mass & \%mass \\
\hline $\mathrm{Cu}$ & 78 & 79 & 78 & $\mathrm{Cu}$ & 70 & 71 & 70 & $\mathrm{Cu}$ & 60 & 62 & 59 \\
\hline As & 0,8 & tr & tr & As & nd & nd & nd & As & nd & nd & nd \\
\hline $\mathrm{Ag}$ & 21 & 20 & 21 & Ag & 30 & 29 & 30 & $\mathrm{Ag}$ & 40 & 38 & 41 \\
\hline & 99,8 & 100 & 100 & & 100 & 100 & 100 & & 100 & 100 & 100 \\
\hline
\end{tabular}

\begin{tabular}{|c|c|c|c|c|c|c|c|c|c|c|c|}
\hline $50 \mathrm{Ag}$ & & & & $60 \mathrm{Ag}$ & & & & $70 \mathrm{Ag}$ & & & \\
\hline Elt & $\%$ mass & $\%$ mass & $\%$ mass & Elt & $\%$ mass & \%mass & \%mass & Elt & $\%$ mass & $\%$ mass & $\%$ mass \\
\hline $\mathrm{Cu}$ & 48 & 46 & 47 & $\mathrm{Cu}$ & 43 & 43 & 42 & $\mathrm{Cu}$ & 29 & 30 & 30 \\
\hline As & tr & tr & tr & As & tr & tr & tr & As & nd & nd & nd \\
\hline Ag & 52 & 54 & 52 & $\mathrm{Ag}$ & 57 & 57 & 57 & Ag & 71 & 70 & 70 \\
\hline & 100 & 100 & 100 & & 100 & 100 & 100 & & 100 & 100 & 100 \\
\hline
\end{tabular}

\begin{tabular}{|c|c|c|c|c|c|c|c|c|c|c|c|}
\hline $80 \mathrm{Ag}$ & & & & $90 \mathrm{Ag}$ & & & & $95 \mathrm{Ag}$ & & & \\
\hline Elt & $\%$ mass & \%mass & $\%$ mass & Elt & $\%$ mass & \%mass & \%mass & Elt & $\%$ mass & \%mass & $\%$ mass \\
\hline $\mathrm{Cu}$ & 19 & 19 & 19 & $\mathrm{Cu}$ & 7,5 & 7,5 & 7,5 & $\mathrm{Cu}$ & 4 & 3,6 & 4 \\
\hline As & 0,8 & 0,7 & 0,6 & As & 0,9 & 0,7 & 1,2 & As & 1 & 1,3 & 1 \\
\hline Ag & 80 & 80 & 80 & $\mathrm{Ag}$ & 91 & 92 & 91 & $\mathbf{A g}$ & 95 & 95 & 95 \\
\hline & 100 & 100 & 100 & & 100 & 100,2 & 99,7 & & 100 & 100 & 100 \\
\hline
\end{tabular}

Fig. 5 : Analyses des alliages. La composition chimique globale a été déterminée par Spectromètrie Dispersive en Energie (EDS) couplée au Microscope Electronique à Balayage (MEB Cambridge Instruments Stereoscan 120) avec une tension d'accélération de $15 \mathrm{kV}$ et durée d'analyse de 120 secondes. Chaque échantillon a été préparé afin de présenter une coupe verticale permettant des dosages depuis la surface de la pièce jusqu'à sa base. $\mathrm{Tr}=\operatorname{trace}$ (moins de $0,5 \%) \mathrm{Nd}=$ non dosé.

garder présent à l'esprit qu'il s'agit d'une information très ponctuelle et qui ne représente ni la température moyenne du moufle, ni celle de la coupelle. Les sondes sont placées de préférence au fond du moufle dans la zone la plus chaude. Une première chauffe à blanc a été faite en plaçant des sondes à l'entrée, au milieu et au fond du moufle. Elle permet d'apprécier les capacités de montée en température du four. Il est possible d'atteindre des températures proches de $1100^{\circ} \mathrm{C}$ alors que l'entrée du moufle avoisine au même moment $900^{\circ} \mathrm{C}$. Lors des mesures en cours d'opération, il est apparu que les conditions climatiques extérieures influent largement sur la température du moufle ${ }^{4}$. D'une manière générale, durant les coupellations, la température au fond du moufle est comprise entre $850^{\circ} \mathrm{C}$ et $950^{\circ} \mathrm{C}$ ce qui est au dessus du point de fusion des oxydes formés. D'autre part, la charge de combustible permet de maintenir sans ajout de charbon la température de travail pendant 30 à 40 minutes (fig. 6). Il s'agit d'une moyenne constatée sur l'ensemble des expérimentations. Les variations dépendent principalement des conditions climatiques externes.

L'essai commence par le préchauffage de la coupelle qui s'effectue soit sur un foyer indépendant du four, soit devant l'ouverture du moufle. Il s'agit de débarrasser le plus complètement possible la coupelle de son eau afin d'éviter qu'elle ne se rompe au cours de l'expérience entraînant l'échec de l'essai. La coupelle est ensuite enfournée dans le moufle. Après ce préchauffage, on introduit la prise d'essai et la porte du four est refermée pour faciliter sa fusion. Une fois la prise liquide, il faut introduire la quantité de plomb nécessaire à l'opération de coupellation. Cet ajout de plomb est directement en relation avec la quantité de cuivre présent. Une table des essayeurs a été établie qui fournit la quantité de plomb à ajouter en fonction du titre estimé de l'alliage testés. Cet ajout est d'autant plus important qu'il y a une forte proportion de cuivre. Pourtant, au-delà d'un alliage à $50 \%$ de cuivre, la masse de plomb à ajouter est donné comme étant systématiquement 16 à 17 fois la masse de la prise. Il s'agit vraisemblablement d'une simplification des essayeurs dans la quantification du plomb pour des alliages à bas titres. Lorsque l'ensemble est fondu, la porte du moufle est ouverte pour permettre la circulation de l'air depuis l'extérieur vers l'intérieur du moufle créant ainsi en permanence une atmosphère oxydante. Il se forme alors à la surface du liquide des taches d'oxydes de plomb et de cuivre qui glissent vers le pourtour du bain. Ces oxydes sont liquides et commencent à imbiber la coupelle. L'opération est facilement perceptible car la couleur de la cendre change du blanc au noir là où s'imbibent les oxydes (fig. 7). A mesure que la coupelle s'imprègne, le niveau du liquide baisse. L'opération est arrêtée à son terme lorsque la production de plaques d'oxydes cesse et que le métal liquide produit une brillance fugace. $\mathrm{Ce}$ bref instant est connu des métallurgistes sous le nom " d'éclair d'argent ». La coupelle est alors retirée progressivement du moufle pour éviter le " rochage ». En effet, l'argent liquide a la capacité d'absorber une grande quantité d'oxygène. Un refroidissement brutal entraîne un dégazage violent de cet oxygène pouvant conduire à des projections d'argent hors de la coupelle ce qui fausse l'essai alors même que le rochage se produit surtout avec de l'argent fin.

Compte tenu de ces données, il est facile de définir les incidents pouvant survenir lors de l'opération. Ils peuvent se résumer en deux grandes familles. La ruine de la coupelle est le phénomène le plus fréquent. En raison d'un mauvais séchage, cette rupture se produit le plus souvent dès l'enfournement de la coupelle. L'objet peut également être fragilisé lors de sa manipulation car il ne se compose que de cendre compactée. Si une fissure apparaît, le métal peut s'y loger et va se dilater lors de la montée en température fragilisant, parfois jusqu'à la rupture, la coupelle. L'autre cause d'échec est connue sous le terme d'ennoyage du bouton. Il se produit lorsque la température baisse inopinément solidifiant les oxydes. Elle est difficilement réversible car pour augmenter la température, il faut recharger en combustible le four ce qui provoque au préalable une chute encore plus brusque de la température. L'ennoyage peut également se produire

\footnotetext{
${ }^{4}$ L'absence de vent rend plus délicat le tirage ce qui a pour conséquence directe de mauvaises montées en température.

s Cette table a été établie par D'Arcet en 1816 (Ann. de Chimie et de Pharm., (Caunedo et Cordoba, 2000), 1, 66, 1816) et a été depuis reproduite à l'identique.
} 


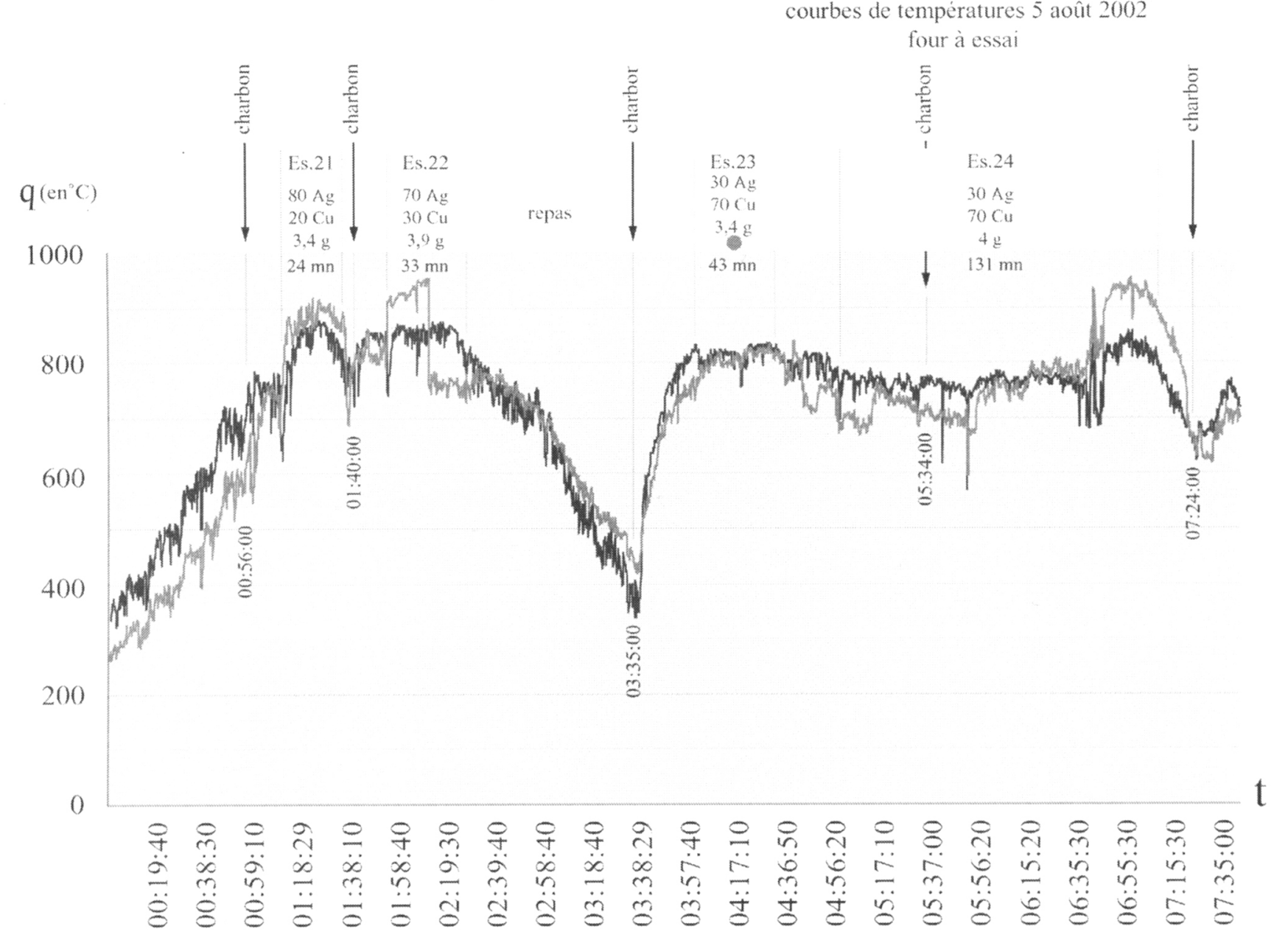

Fig. 6 : Courbes de température (en noir, mesuré au fond du moufle, en gris au milieu)

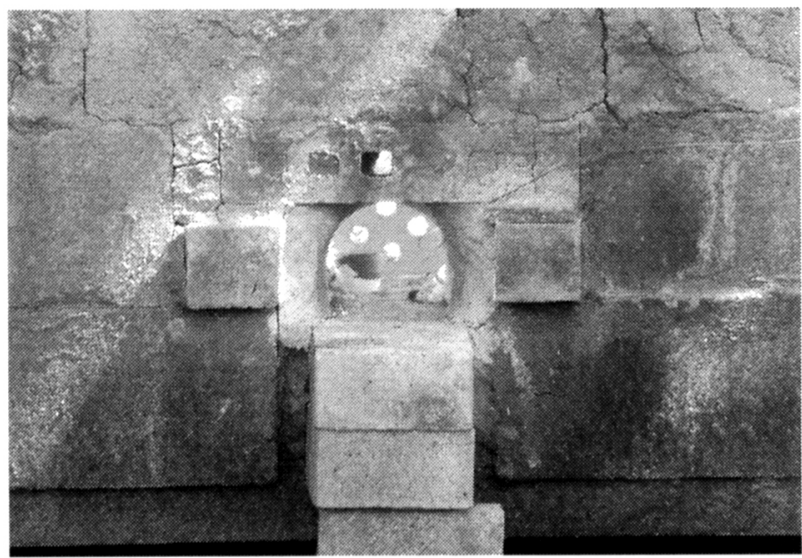

Fig. 7 : Une coupellation en cours.

lorsque la coupelle est totalement imbibée. Dans chaque cas, le bouton se couvre d'un voile d'oxydes protecteurs et l'opération ne peut aboutir car il est techniquement très difficile de remonter au point de fusion des oxydes.

\section{RÉSULTAT ET DISCUSSION}

L'appréciation de la qualité des coupellations effectuées expérimentalement s'est faite selon un mode empirique qui consiste à peser la prise de départ puis le bouton de retour en argent pur. Le rapport entre ces deux données fournit le pourcentage d'argent présent dans l'alliage. Sur les 22 coupellations cuivre-argent tentées, 15 ont été considérées comme réussies. C'est-à-dire que la coupelle ne s'est pas rompue et que le bouton ne s'est pas noyé dans la litharge. Dans certains cas, il a été possible de percevoir l'éclair. Les boutons rochés sont comptés comme réussis. Parmi cet ensemble, neuf sont choisis permettant de couvrir l'ensemble de la gamme des alliages testés (fig. 8). Il en résulte systématiquement une différence entre la masse théorique du bouton et la masse constatée. Cette différence s'inscrit dans une fourchette $\mathrm{de}+/-4 \%$. Les boutons sont généralement d'une masse inférieure. Cette différence peut être imputée pour une bonne part au déroulement de la manipulation ${ }^{6}$. Si l'on tient compte des analyses des alliages qui montrent des taux d'argent différents de ceux attendus, alors les variations sur la masse des boutons de retour s'inscrivent entre $-5 \%$ et $+2 \%$ des masses prévues. Il semble que de façon générale l'on tende plus à sous-estimer la quantité d'argent présent.

Les variations de titre mises en évidence aussi bien par les mesures de masse des boutons que par comparaison avec la nature des alliages incitent à déterminer par analyse quantitative la teneur en argent de ces boutons. Il n'est pas inutile d'observer au préalable la composition des 


\begin{tabular}{|c|c|c|c|c|c|c|c|c|c|}
\hline $\begin{array}{c}\mathrm{n}^{\circ} \\
\text { d'essai }\end{array}$ & \begin{tabular}{|c|} 
Alliage \\
$\mathrm{Ag} / \mathrm{Cu}$ \\
Titre \\
estimé
\end{tabular} & $\begin{array}{c}\text { masse } \\
\text { de la } \\
\text { prise } \\
\text { en g }\end{array}$ & $\begin{array}{l}\text { masse } \\
\text { de } \\
\text { plomb } \\
\text { ajouté } \\
\text { en g }\end{array}$ & $\begin{array}{c}\text { Masse du } \\
\text { bouton } \\
\text { en } g\end{array}$ & $\begin{array}{c}\text { Masse } \\
\text { théorique } \\
\text { du bouton } \\
\text { en } g\end{array}$ & $\begin{array}{l}\text { différence } \\
\text { masse réel / } \\
\text { masse } \\
\text { théorique }\end{array}$ & $\begin{array}{c}\text { titre estimé } \\
\text { des alliages à } \\
\text { partir des masse } \\
\text { des boutons } \\
\text { (le bouton est } \\
\text { supposé en } \\
\text { argent pur) }\end{array}$ & $\begin{array}{c}\text { Titre de } \\
\text { l'alliage, } \\
\text { analyse } \\
\text { EDS }\end{array}$ & \begin{tabular}{|c|} 
différence \\
masse réel \\
/ masse \\
théorique \\
en fonction \\
de \\
l'analyse \\
EDS de \\
l'alliage de \\
départ \\
\end{tabular} \\
\hline 1 & $95 / 5$ & 7 & 21 & 6.36 & 6.65 & $-4 \%$ & $91 \%$ & $95 \%$ & $-4 \%$ \\
\hline 30 & \begin{tabular}{|c|c|}
$90 / 10$ \\
\end{tabular} & 2 & 14 & 1.74 & 1.8 & $-3 \%$ & $87 \%$ & $91.5 \%$ & $-5 \%$ \\
\hline 31 & \begin{tabular}{|l|}
$80 / 20$ \\
\end{tabular} & 1.7 & 17 & 1.32 & 1.36 & $-3 \%$ & $77 \%$ & $79.7 \%$ & $-2 \%$ \\
\hline 22 & $70 / 30$ & 3.9 & 46.8 & n.d. & 2.73 & n.d. & n.d. & $70.1 \%$ & n.d. \\
\hline 42 & $60 / 40$ & 2.1 & 29.4 & 1.22 & 1.26 & $-3 \%$ & $58 \%$ & $56.8 \%$ & $+2 \%$ \\
\hline 43 & \begin{tabular}{|l}
$50 / 50$ \\
\end{tabular} & 2.3 & 36.8 & 1.2 & 1.15 & $4 \%$ & $52 \%$ & $52.7 \%$ & $-1 \%$ \\
\hline 46 & $30 / 70$ & 3.9 & 66.3 & 1 & 1.17 & $-14 \%$ & $25 \%$ & 29.6 & -15 \\
\hline 17 & $20 / 80$ & 1.7 & 28.9 & 0.35 & 0.34 & $3 \%$ & $20,5 \%$ & $20.8 \%$ & $-1 \%$ \\
\hline 47 & \begin{tabular}{|l}
$20 / 80$ \\
\end{tabular} & 2.8 & 47.6 & 0.5 & 0.56 & $-10 \%$ & $18 \%$ & $20.8 \%$ & $-16 \%$ \\
\hline
\end{tabular}

Fig. 8 : Tableau des 9 coupellations.

métaux ayant servi dans la composition des alliages. Si le plomb peut être considéré comme pur (les traces de silicium et d'aluminium qui apparaissent sont imputables à une pollution lors du polissage), il n'en va pas de même pour le cuivre qui contient des traces d'argent et de plomb. L'argent, quant à lui, contient un pourcentage non négligeable d'arsenic compris entre $0.5 \%$ et $1 \%$. Ici encore la composition chimique globale en pourcentage massique a été déterminé par EDS couplée au MEB avec une tension d'accélération de $15 \mathrm{kV}$.
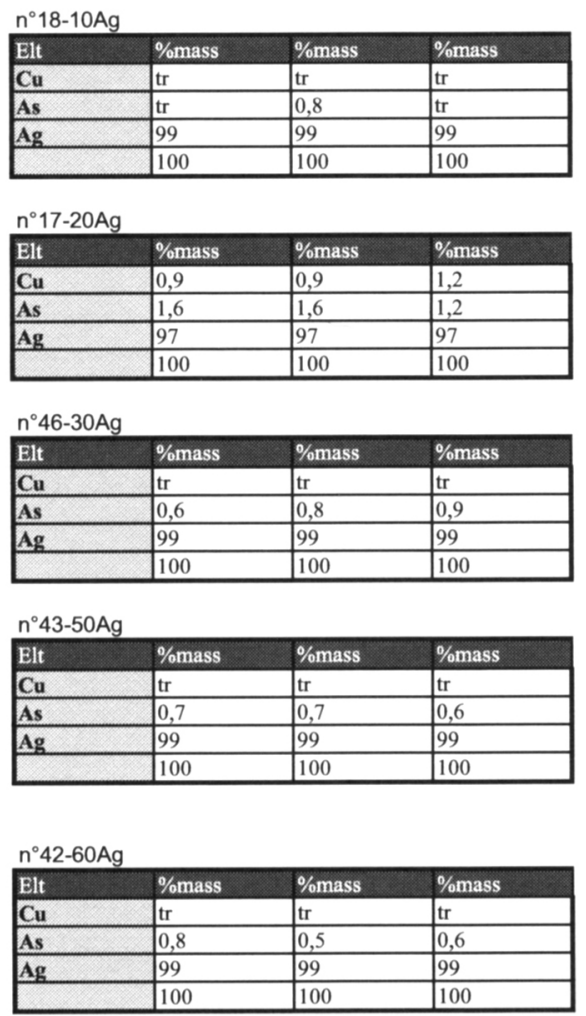

Les boutons de retour ont été sciés en deux permettant des analyses à différentes épaisseurs (surface, milieu et fond) pour mieux apprécier les variations de teneurs et donner une valeur globale représentative de la pièce. Les résultats sont remarquables par leur régularité (fig. 9). Les boutons d'argent contiennent en moyenne entre $98 \%$ et $99 \%$ d'argent quelque soit l'alliage de départ. L'arsenic qui avait été vu systématiquement dans les granules d'argent se retrouve dans des proportions équivalentes ou inférieures. On peut donc considérer que dans la majo-
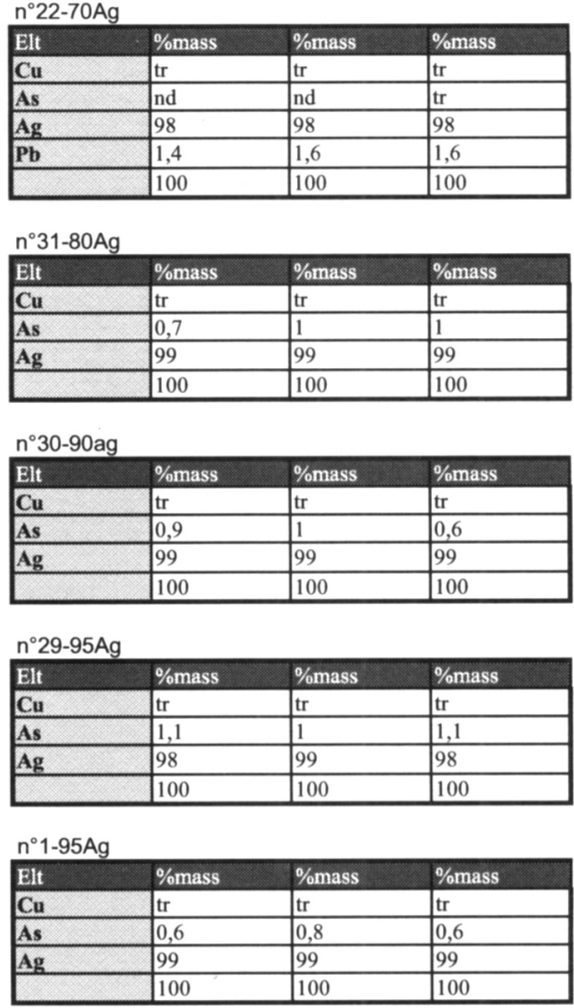

Fig. 9 : Analyse des boutons de retour. La composition chimique globale a été déterminée par Spectromètrie Dispersive en Energie (EDS) couplée au Microscope Electronique à Balayage (MEB Cambridge Instruments Stereoscan 120) avec une tension d'accélération de 15 kV et durée d'analyse de 120 secondes, carbone non dosé. $\mathrm{Tr}=$ trace (moins de $0,5 \%) \mathrm{Nd}=$ non dosé. 
rité des cas, il a été possible de retrouver l'argent d'origine. Le plomb n'est visible que dans un seul bouton (essai $\mathrm{n}^{\circ} 22$ avec un alliage à $70 \%$ d'argent). Dans tous les autres cas, il n'a pu être détecté. La présence de ce métal dans le bouton de retour est intéressante car lors de l'expérience il a été possible de voir sur cet essai l'éclair d'argent qui caractérise habituellement la fin de l'opération et son succès. Enfin le cuivre n'est jamais totalement oxydé. Il est toujours présent à l'état de trace (moins de $0.5 \%$ ) sauf dans un cas où le taux atteint $1.5 \%$ (coupellation $n^{\circ} 17$ ). Là encore, bien que le bouton contienne un pourcentage non négligeable de métaux alliés, il a été possible de voir l'éclair d'argent. Cet éclair n'est donc pas nécessairement synonyme d'obtention d'un argent pur comme on peut le lire dans la plupart des ouvrages métallurgiques depuis Agricola et Biringuccio. En revanche, il s'agit bien d'un repère qui marque un bon niveau de pureté de l'argent.

Disposant de coupelles expérimentales usagées, il est intéressant d'observer de quelle façon s'est faite la diffusion des oxydes au sein de l'objet. Cette approche archéométrique permet de pallier aux difficultés d'accès à des coupelles archéologiques tout en facilitant à terme leur étude. Deux coupelles ont été préparées pour analyses. Il s'agit des coupelles 22 et 47 qui représentent des extrêmes entre un alliage bas titre pour la coupelle $47(20 \% \mathrm{Ag})$ et un alliage bon titre pour la coupelle $22(70 \% \mathrm{Ag})$. Sur

\section{Coupelle $n^{\circ} 22$}

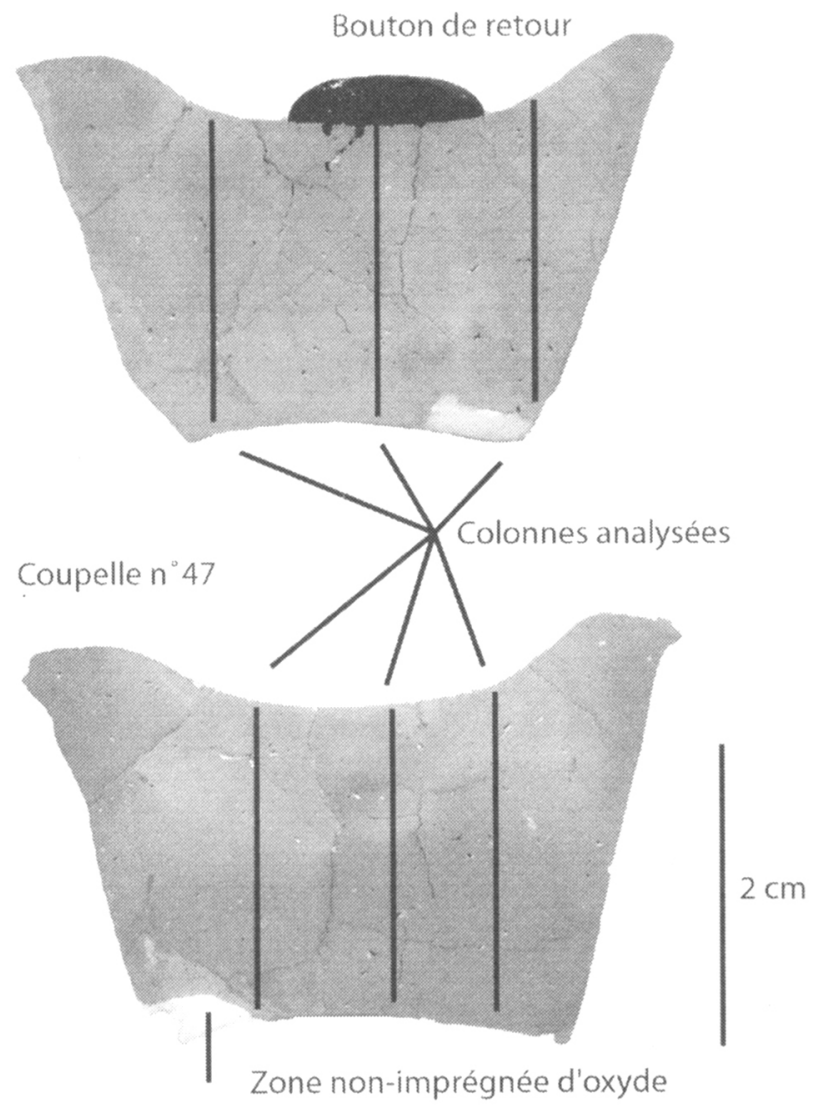

Fig. 10 : Vue macroscopique des coupelles 22 et 47 montrant les segments analysés. une coupe transversale, une série d'analyses ponctuelles $\left(6 \mathrm{~mm}^{2}\right)$ est faite en colonne afin d'obtenir une image de la répartition des différents éléments et de leur évolution à mesure que l'on progresse vers la base de la coupelle (fig. 10). Quatre rapports sont établis : $(\mathrm{P}+\mathrm{Ca}) / \mathrm{Cu}$, $(\mathrm{P}+\mathrm{Ca}) / \mathrm{Pb}, \mathrm{Cu} / \mathrm{Pb}$ et $\mathrm{Ag} / \mathrm{Pb}$ (fig. 11). Le phosphore et le calcium sont les deux constituants principaux de la coupelle avant son utilisation ${ }^{7}$. Le plomb, le cuivre et l'argent représentent les trois métaux principaux qu'il est possible de retrouver après utilisation. Le rapport $(\mathrm{P}+\mathrm{Ca}) / \mathrm{Pb}$ montre dans les deux cas une constante qui s'établit autour de 0,6. L'oxyde de plomb diffuse donc de façon homogène dans l'ensemble du matériau. À l'inverse, le rapport $(\mathrm{P}+\mathrm{Ca}) / \mathrm{Cu}$ met en lumière un accroissement de la présence du cuivre vers la base de la coupelle. Ce rapport décroissant du sommet vers la base se stabilise entre $9 \mathrm{~mm}$ et $12 \mathrm{~mm}$ de profondeur dans les deux coupelles 47 et 22 . Ce phénomène pourrait être le reflet de l'absorption préférentielle de l'oxyde de cuivre en début de coupellation mais le phénomène n'est, pour l'heure, pas clairement défini. Le troisième rapport $\mathrm{Cu} / \mathrm{Pb}$ est conforme aux résultats trouvés précédemment à savoir que la concentration du cuivre par rapport au plomb est stable jusqu'à $9 \mathrm{~mm}$ de profondeur puis augmente régulièrement dans le cas de la coupelle 22 et brutalement dans le cas de la coupelle 47. Cette variation d'intensité tient à la quantité de cuivre présent dans l'alliage de départ ( $30 \%$ dans un cas, $80 \%$ dans l'autre). Enfin, il importe de savoir si cette coupellation entraîne une perte d'argent. La présence d'une petite quantité d'argent passée dans la coupelle est connue des alchimistes qui broyaient les coupelles usagées pour en retirer l'argent par descension dans un creuset à fond percé (Vigenère, 1618). Cette perte existe bel et bien puisque le taux d'argent rencontré s'établit systématiquement à plus de $1 \%$. Les profils réalisés dans les coupelles mettent en évidence une bonne corrélation entre le plomb et l'argent. Il apparaît surtout que le rapport est identique pour les deux coupelles analysées alors que les rapports $\mathrm{Ag} / \mathrm{Pb}$ de départ varient d'un facteur 5. On peut en conclure que la perte d'argent en masse est la même quelque soit le rapport de départ. Ainsi il semble bien que cette perte soit proportionnellement d'autant plus importante que l'alliage est faible en argent. Il s'agit peut être là d'une des raisons de l'abandon de ce procédé, au moins au sein des ateliers monétaires, car il ne permet pas de doser avec suffisamment de précision ces alliages bas titre qui constituent une masse monétaire très importante (Calmels, 2002). D'autre part, la réalisation de ces profils verticaux permet également de voir que les oxydes diffusent de façon uniforme sur le plan horizontal. La coupelle joue bien ici le rôle d'une " éponge " puisque l'imbibition se fait par capillarité jusqu'à son sommet, bien au-delà du niveau premier du bain métallique. Les variations apparaissant entre les colonnes dans les différents rapports ne sont probablement que le reflet des conditions externes d'expérimentation et marquent principalement la circulation des gaz dans le moufle ainsi que la position plus ou moins horizontale de l'objet lors de la coupellation. 

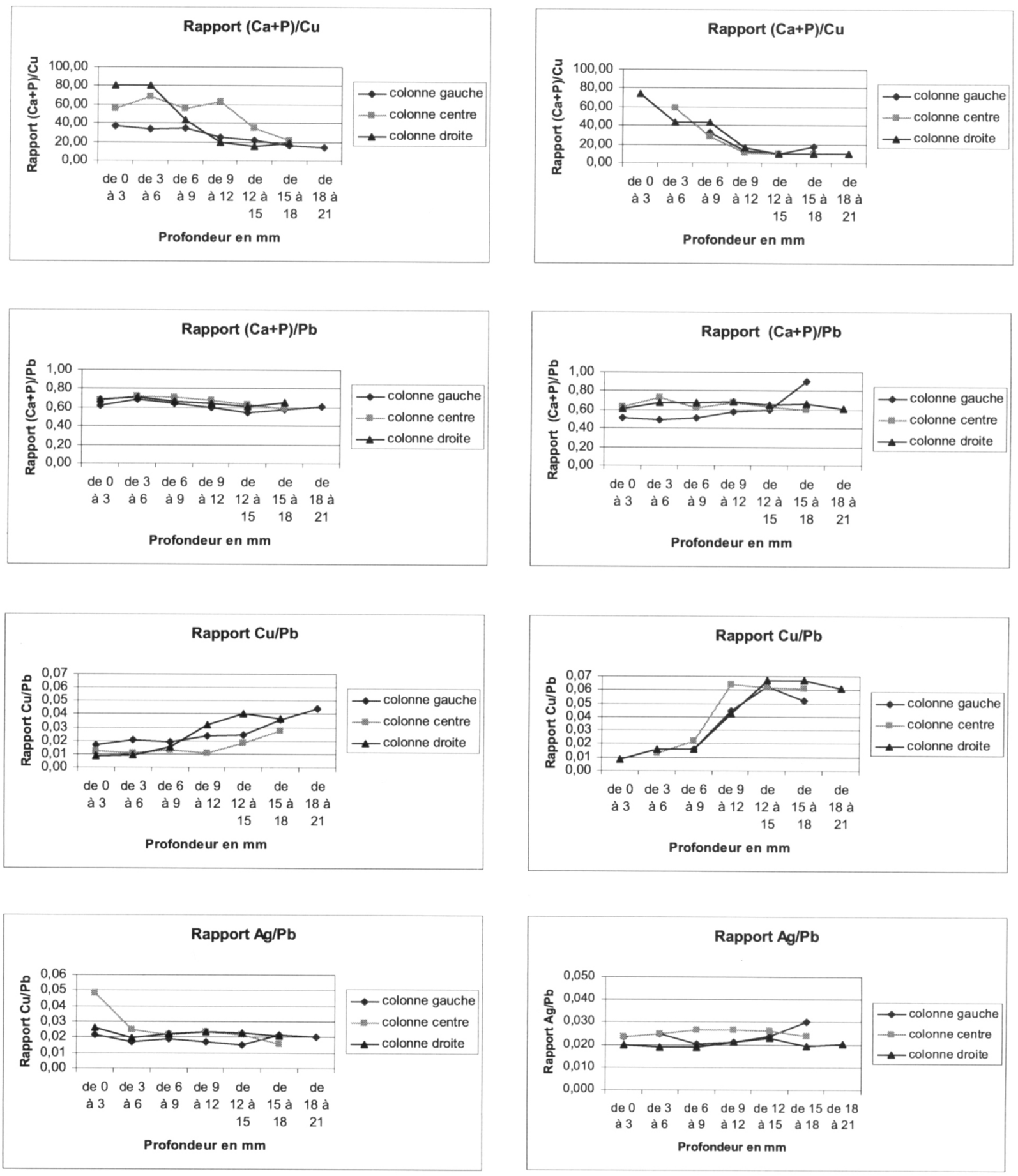

Fig. 11 : Rapports des éléments dans les coupelles 22 et 47 (composition chimique globale en pourcentage massique, EDS couplée au MEB, $15 \mathrm{kV}$, carbone non dosé).

\section{CONCLUSION}

L'essai par voie sèche a fait l'objet d'une abondante littérature chimique bien après son abandon dans les ateliers monétaires. Les précis de Riche comme celui de Campredon en sont sûrement les formes les plus abouties. Ils sont publiés au tournant du XIX ${ }^{\mathrm{e}}$ siècle et ne font pas mention des difficultés rencontrées pour la fabrication monétaire bien que la table des essayeurs fournit par D'Arcet soit publiée par tous ces auteurs. Il est évident que pour les alliages cuivre-argent, la technique d'essai par voie sèche est bien abandonnée dès le $2^{\mathrm{e}}$ tiers du $\mathrm{XIX}^{\mathrm{e}}$ siècle au profit du dosage par attaque acide mis au point par Gay-Lussac (Gay-Lussac, 1832). L'essai par voie sèche reste cependant en usage encore de nos jours puisqu'il est possible de le rencontrer dans les laboratoires des essayeurs aussi bien en Bolivie qu'au Maroc. L'utilisation actuelle d'un procédé somme toute moins précis que l'essai par voie humide se comprend par sa grande facilité de mise en œuvre, son moindre coût et sa plus grande rapidité d'exécution (Fresenius, 1900). Cette approche archéologique expérimentale complète les tra- 
vaux menés sur les essais par voie sèche. Elle permet de mieux comprendre les causes de l'abandon de cette coupellation à la cendre au sein des ateliers monétaires. En suivant les travaux de d'Arcet, seul l'hétérogénéité des alliages est mise en cause. Or il faut également tenir compte de la perte d'argent dans la coupelle. Cette perte n'est, semble-t-il, pas comptabilisé au sein des ateliers avant une date tardive. Riche qui écrit son traité à la fin du XIX ${ }^{\mathrm{e}}$ siècle donne pourtant une table de compensation. Or cette perte est bien perçue dans les usines métallurgiques où les coupelles de cendre servant au raffinage de l'argent sont récupérées pour être retraitées (Archives de l'Ecole des Mines de Paris).

\section{BIBLIOGRAPHIE}

AGRICOLA, G., 1987 - De Re Metallica, Bâle, 1556, France-Lannord éd., Thionville, 203-204.

Archives de l'Ecole des Mines de Paris, M 1834 (167), Regnault M. V., Mémoire sur les mines et usines de Clausthal au Hartz, 1834, p. 22 .

BERTHIER, M. P., 1834 - Traité des essais par la voie sèche ou des propriétés, de la composition et de l'essai des substances métalliques et des combustibles, Tome second, Paris, Thomine libraire, 1834, 2 vol., 654 p. et $1008 \mathrm{p}$.

CALMELS, L., 2002 - La précision des essais d'argent remise en question, les papiers d'Arcet à la monnaie de Paris, Revue Numismatique, 5-14.

CAMPREDON, L., 1909 - Guide pratique du chimiste métallurgiste et de l'essayeur, $2^{\mathrm{c}}$ édition, Paris, art. 828, 303-304.

CAUNEDO, B. \& CORDOBA, R., 2000 - El arte del Alguarismo, Valladolid, Junta de Castille et Léon, 217-223.

CLAIRAND, A. et KIND, J.-Y., 2000 - Le traité des monnaies de Jean Boizard d'après l'édition de Paris de 1692, (Documents d'Histoire Monétaire, Sources imprimées, 1), K\&C éd., 85-88.

CORBETT, J., 1939 - Catalogue des Manuscrits Alchimiques Latins, Manuscrits des bibliothèques publiques de Paris antérieurs au XVIT siècle, Union Académique Internationale, Bruxelles, p. 91.

FRESENIUS, R., 1900 - Traité d'analyse chimique quantitative, $7^{\mathrm{e}}$ édition française traduite par L. Gautier, Paris, Masson et $\mathrm{C}^{\mathrm{ie}}$ éditeurs, p. 971.

GAY-LUSSAC, L.-J., 1832 - Instruction sur les essais des matières d'argent par la voie humide, commission des Monnaies et Médailles, Paris, Imprimerie Royale, 63 p.

GOLDENBERG, G. et WAGNER, H., 1994 - La métallurgie des non-ferreux dans la Forêt Noire du Moyen Age au XIX ${ }^{\mathrm{e}}$ siècle, in
Benoit P., (dir.), Mines et métallurgie, coll. Les chemins de la recherche, Programme Pluriannuel en Sciences Humaines Rhône-Alpes, 21, Lyon, p. 69.

GOY, C. et THOMAS, N., 2003, à paraître - Les fouilles du bourg Saint-Martin - distillation et coupellation, deux opérations alchimiques dévoilées, in Bulletin de la Société d'Émulation de Montbéliard.

HALLEUX, R., 1981 - Les alchimistes grecs, Papyrus de Leyde, Papyrus de Stockholm, Recettes, Tome I, Les Belles Lettres, Paris, p. 91.

HOEFER, F., 1866-1869 - Histoire de la chimie depuis les temps les plus reculés jusqu'à notre époque, Paris, 1842-1843 ; 2ème édition, Firmin Didot, Paris, vol. 1, 499-500.

JEANJACQUOT, J.-C. et GOY, C., 1993 - Pymont, la forteresse oubliée - XIII $-X V^{e}$ siècle, entre les Vienne et les Chalon, Lons-leSaulnier, p. $97 \mathrm{sq}$.

NEWMAN, W. R., 1991 - The Summa perfectionis of Pseudo-Geber. A critical edition, translation and study, Collection de travaux de l'Académie internationale d'histoire des sciences, t. XXXV, E. J. Brill, Leiden, New York, København, Köln, p. 590 sqq.

REHREN, T., 1996 - Alchemy and fire assay - An analytical approach, in JHMS, 30, 2, 136-142.

REHREN, T., 1997 - Metal analysis in the Middles Ages, in Deboe G., Verhaeghe F., Material Culture in Medieval Europe, Papers of the Medieval Europe Brugge 1997' Conference, Vol. 7, Zellik, 9-15.

REHREN, T., 1998 - Kontext und Analyse der Aschkupellen von Oberstockstall, in Osten S. von, Das Alchemistenlaboratorium Oberstockstall, Ein Fundkomplex des 16. Jahrunderts aus Niederösterreich, Monographien zur Frühgeschichte und Mittelalterarchäeologie, Wagner, Innsbruck, 333-348.

RICHE, A. et GELIS, E., 1888 - L'art de l'essayeur, bibliothèque des connaissances utiles, librairie J.B. Baillières et fils, Paris, 384 p.

SLOTTA, R., WOLLMANN, V. \& DORDEA, I., 1999 - Silber und salz in Siebenbürgen, Katalog zur Ausstellung im Deutschen Bergbau-Museum vom 27. August bis zum 31. December 2000, tome 1, Bochum, 334-335.

TÉREYGEOL, F. et THOMAS, N., à paraître - L'essai d'argent expérimentations selon les recettes antiques et médiévales, Actes du colloque forgerons et orfêvres tenu à Toulouse en 2001.

THÉOPHILE, 1977 - Essai sur divers arts, diversarum artium schedula, publié et traduit par le $\mathrm{c}^{\text {te }}$ Escalopier, J. Laget, P. Daviaud, Librairie des Arts et Métiers, Nogent-le-Roi, chap. XII, p. 143, chap. LXVIII, p. 229, chap. LXIX, p. 231.

TOLL, C., 1968 - Al-Hamdani, Kitab al-Gauharatain Al-'aliqatain AlMâ'i'atain As-Safrâ' Wa't Barda, Die beiden Edelmetalle Gold und Silber hrsg. U. übers von C. Toll, Acta Univ. Upsala, studia Semetica Upsaliensa, 1, Upsala, 274-284.

VIGENÈRE, B. (de), 1618 - Traicté du Feu et et du Sel, Excellent et rare Opuscule du sieur Blaise de Vigenère, Bourbonnois, trouve parmy ses papiers après son décés. Abel l'Angelier, Paris, 86-87. 